\title{
The Design of Combined DCU (Data Concentration Unit) System with Power-Line Communication and DOCSIS Cable Modem for AMR (Auto Meter Reading)
}

\author{
Su-Mi Jeong ${ }^{1, ~ a ~}$, Chung-Ki Seo ${ }^{1, b}$ and Sang-Jin $\mathrm{Kim}^{2, \mathrm{c}}$ \\ ${ }^{1}$ Dept. of Embedded Research, KEPCO KDN, \\ Naeson 2-Dong, Uiwang-Si, Gyeonggi-Do 437-715, Korea \\ ${ }^{2}$ R\&D Department Associate, KEPCO KDN, \\ Naeson 2-Dong, Uiwang-Si, Gyeonggi-Do 437-715, Korea \\ asm3010@kdn.com, ${ }^{\mathrm{b}} \mathrm{ckseo@kdn.com,}{ }^{\mathrm{c}} \mathrm{sjkim@kdn.com}$
}

Keywords: smart grid, advanced metering infrastructure, auto meter reading, data concentrate unit, power line communication, cable modem communication.

\begin{abstract}
The AMR (Auto Meter Reading) is needs to prepare separate IPs and communication lines for remote communication because DCU (Data Concentration Unit) and wideband modem are identical system required the resource to communication. It is the purpose of the literature to improve system stability and data reliability by means of integrating broadband cable communication part and PLC communication part within DCU. A PLC has some draw-backs in wideband communication because of absences of international standard specifications and the characteristic of power line. We integrated broadband cable modem and PLC modem in DCU. Therefore, the communication system is designed with unified board. PLC modem and broadband cable modem are designed to meet ISO/IEC12139-1 and international specified DOCSIS2.0 standard, respectively.
\end{abstract}

\section{Introduction}

The AMI is core technology for establishing the smart grid and it is also base technology for the comprehensive energy management system based on the bi-directional communication that will substitute the present closed energy supply system based on the uni-directional communication.[1] The AMI provides real-time energy consumption information to consumers and the consumers can reduce their home and company energy cost by themselves or by controlling automated equipments. Utilities can reduce the maximum output during peak time by DR(Demand Response) and load control as well as they can save the cost of meter reading and maintenance. Therefore, it is expected to reduce the cost of enenrgy generation and additional infrastructure establishment. Consequently, governments around the world have been actively reviewing the introduction of AMI as part of the protection of environment and conservation of energy resources.[2] In Korea, establishing the AMI system and providing its services are actively in progress mainly with AMR. As well as, the test bed for introducing value-added services has been built and operated.[8]

\section{The AMR-based AMI System Architecture}

At present, there are several kinds of transmission medium in residential network, such as coaxial-cable, radio, microwave, millimeter wave, power line and fiber optics. Compared with other kinds of transmission medium, power line has distinct advantage in setting up a network without additional line installation and existing digital devices, including home appliances and information devices, at very low cost. On the other hand, device power in home automation still can be supplied by power line itself. Therefore, PLC rapidly becomes a popular solution to set up residential network. [3] The cable modems and optic LAN are mainly used for the broadband, rather than PLC because of technical constraints and administrative restrictions. However, in Korea, the KEPCO(Korea Electric Power Coporation) consortium adopts and uses CATV network and PLC communication technology 
as the base communication technology for AMR services. As shown in Fig. 1, the AMR-based AMI system that the KEPCO consortium has developed and provided the service. The metering data is collected from smart meters and this data is transmitted to the E-type PLC modem that transmits it to the DCU and remote server. And this AMI system consists of three parts of infrastructure, DCU that controls sub-devices including smart meters, IHD(InHome Display) that displays real-time AMR information and a metering server for data storage and integrated operation. Based on this infrastructure, it provides bi-directional intelligent power grid services such as real-time enenrgy metering, real-time billing, connected home network information and system control between consumers and ESP(Energy Service Provider).[3]

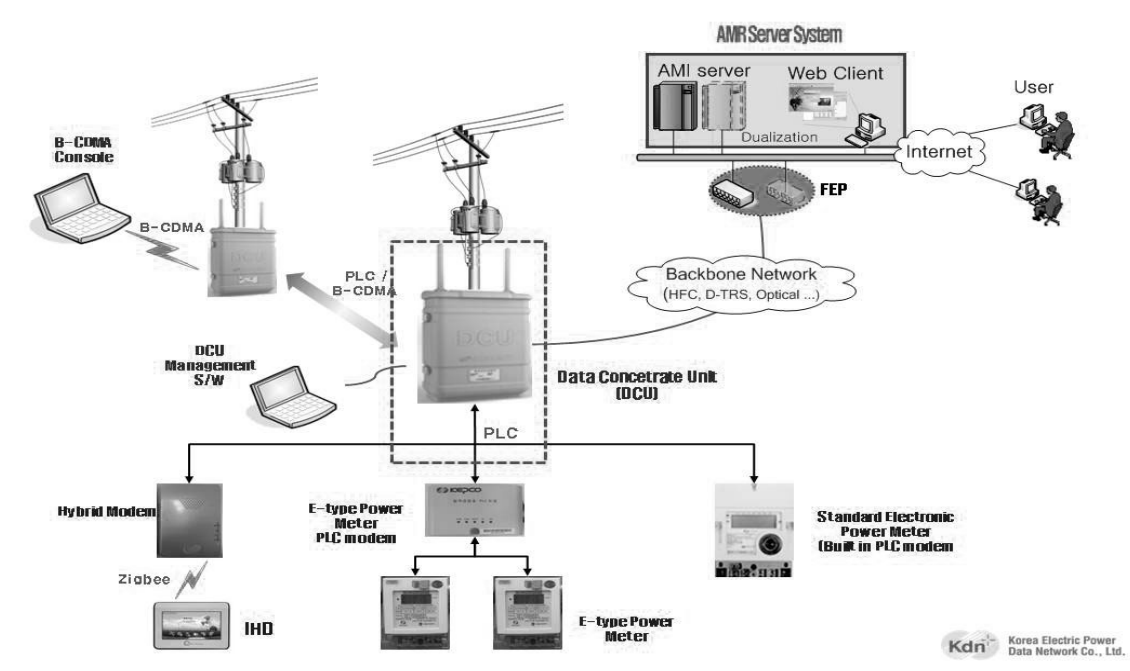

Fig. 1 The AMI configuration

\section{The present DCU System Architecture}

The DCU is the most critical center equipment in the AMI services configuration based on the AMR. A consumer terminal such as smart meter, HM(Hybrid Modem) and IHD is connected to it by PLC and it is connected to the remote server of utilities by CATV network. As a result, the DCU transmits data in bi-directional communication and stores data and controls devices.

The DCU device currently being operated and its block diagram is shown in Fig. 2.

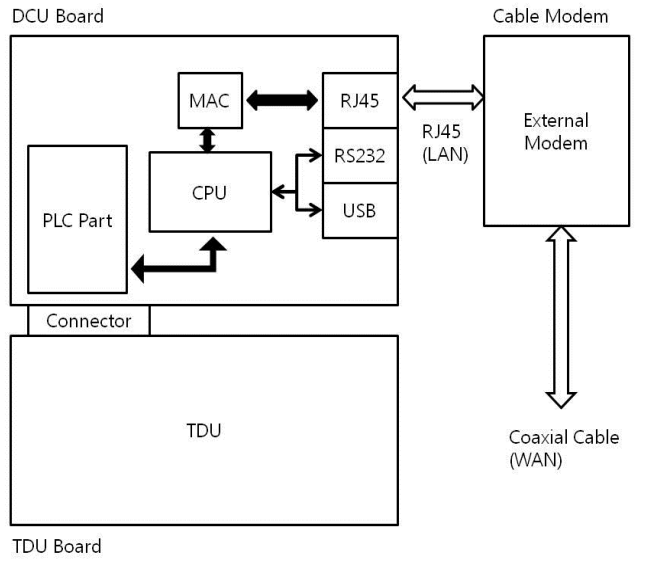

Fig. 2 The built DCU device

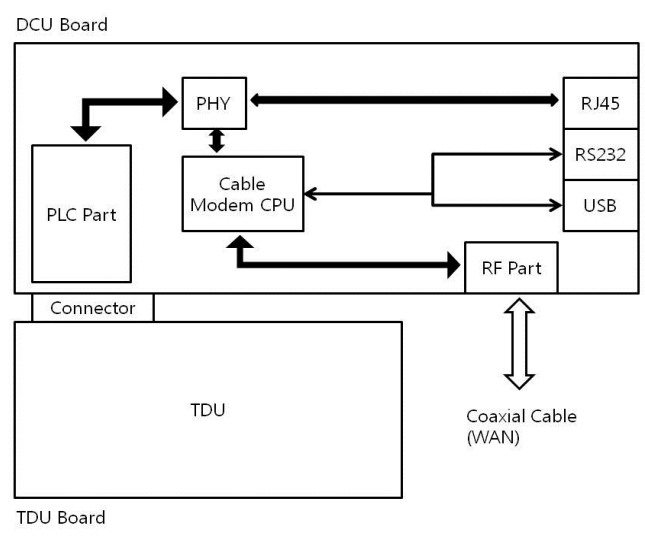

Fig. 3 The cable modem-combined DCU device

The stability and reliability of the DCU device should be ensured absolutely to implement the AMI and provide its services successfully. Because the currently installed DCU has the separate cable modem supporting PLC and cable communication for communication duality, the physical interface between heterogenous devices is necessary and it increases data transmission errors then decreases the data reliability consequentially. In addition, the ethernet is used for the communication between 
heterogenous devices. Therefore it wastes IP address resources and reduces performance because of unnecessary data transformation steps. Most systems use SNMP standard for system management. However it is practically inconvenient to integrate systems and manage devices, because PLC, DCU main part and the separate cable modem use different SNMP respectively.

\section{The proposed cable modem-combined DCU System}

As shown in Figure 3, we developed the cable modem-combined DCU to solve the problems of present DCU.

Because this DCU has the CPU mounted the chip for cable modem-only, we reduced H/W devices, such as CPU and memory and we designed the system concisely by integrating the PLC modem chip.

As well as, we removed the physical interface for heterogeneous devices and reduced the Ethernet IP from 3 to 2 . And integrated management is possible by reducing the MIB configuration for SNMP from three parts into two parts. In addition, we use the cable modem chip as a main cpu of the DCU. As a result, the design of PLC and broadband cable modem meets ISO/IEC12139-1[4] and international specified DOCSIS2.0 standard[5], respectively.[6] Table 1. shows the specification of present DCU and the cable modem combined DCU.

Table. 1 The specification comparison between DCU devices

\begin{tabular}{lll}
\hline Item & Current version & Proposed version \\
\hline CPU & $2[D C U$, Modem] & $1[D C U]$ \\
MAC chip & 1 & 0 \\
PHY chip & 0 & 1 \\
WAN Interface & $2[\mathrm{RJ} 45]$ & $2[$ Coaxial, RJ45] \\
Total IP & $2[\mathrm{DCU}$, Modem] & $1[\mathrm{DCU}]$ \\
SNMP MIB & $3[\mathrm{DCU}$, Modem, PLC] & $2[\mathrm{DCU}, \mathrm{PLC}]$ \\
\hline
\end{tabular}

\section{Conclusion}

A reliability of metered data and stability of the system has important roles in AMR service. The PLC modem is not reasonable choice in broadband communication because of architecture and characteristics of power line. The system can sustain efficient metering and control by integrating PLC modem and cable modem in one board. The PLC modem and Cable modem comply with international standards. Therefore it improved stability of the system and reliability of acquired data, dramatically.References. If you need additional information about smart grid and AMI, please visit our website. [7]

\section{Reference}

[1] Yong-Hee Jeon, Security characteristics and requirements analysis in the zigbee-based AMI, Korea Information Security Institute

[2] ETRI, ETRI, Electronic Communication Trend Analysis, Issue 5, Article 23 The Convergence of Electric Power Technology and USN Technology : A Trend Analysis of USN Based AMI Service and Technology. (2008) 68.

[3] Park, B.S, Hyun, D.H, Cho, S.K. "Implementation of AMR system using power communication". Transmission and Distribution Conference and Exhibition 2002 : Asia Pacific. IEEE/PES, Vol. 1, Oct. 2002, 18-21

[4] Intemational Organization for Standardization, Information technology - and information exchange between systems - Powerline communication (PLC) - High speed PLC medium access control (MAC) and physical layer (PHY), ISO/IEC 12139-1, 2009

[5] ITU-T, Data Over Cable Service Interface Specifications, DOCSIS2.0/3.0, 2010

[6] General Technical Specifications of KEPCO, Data Concentration Unit for Low Voltage system. 2011

[7] Information on http://www.kdn.com

[8] Information on http://smartgrid.jeju.go.kr 